\title{
Loop ileostomy and colonic lavage for fulminant Clostridium difficile colitis: survey of current practice
} \author{
Ann Vasilevsky ${ }^{1}$, Nancy Morin ${ }^{1}$, Julio Faria1, Philip H. Gordon¹, Terry Phang ${ }^{4}$, Marylise \\ Boutros $^{1}$ \\ ${ }^{1}$ Division of Colon and Rectal Surgery, Sir Mortimer B. Davis Jewish General Hospital, Montreal, QC \\ ${ }^{2}$ Department of Colorectal Surgery, Cleveland Clinic Florida, Weston, FL \\ ${ }^{3}$ Division of Colon and Rectal Surgery, University of Minnesota, Minneapolis, MN \\ ${ }^{4}$ St Paul's Hospital, University of British Columbia, Vancouver, BC
}

Maria Abou Khalil1, Steven D. Wexner², Michael P. Spencer ${ }^{3}$, Gabriela Ghitulescu', Carol-

\section{Introduction}

- The gold standard treatment for fulminant Clostrdium difficile colitis (FCDC) is a total abdominal colectomy (TAC)

- Despite this intervention, associated morbidity and mortality remain elevated.

- Diverting loop ileostomy and colonic lavage (LICL) has emerged as an attractive option, but limited data exists.

- We sought to assess surgeons' experience with the use of LICL

\section{Methods}

After IRB approval, a 25 multiple-choice question survey was distributed from 09/2015 to 08/2017 through social media platforms and societal distribution to members of:

- Canadian Association of General Surgeons

- Québec Association of General Surgeons

- International Society of Colon and Rectal Surgeons

- Members of the Society of American Gastrointestinal and Endoscopic Surgeons research committee

- Members of the Colorectal Surgical Society of Australia and New Zealand

\section{Results}

- 135 respondents from Canada (65.4\%), USA (6.8\%), Australia and New Zealand (18.1\%) and 9.7\% from Asia, Europe, Africa and South America

- $17.1 \%$ have done at least $1 \mathrm{LICL}$

- $12.5 \%$ report failure of LICL requiring TAC

- $23 \%$ report at least one recurrence of $C$. difficile infection after LICL and ileostomy closure

- $78.9 \%$ foresee LICL to be indicated for FCDC

- $3.8 \%$ who have not performed a LICL stated more evidence was needed

- $63.5 \%$ were interested in participating in a prospective trial

\section{Results}

Figure 1: Type and volume of lavage administered
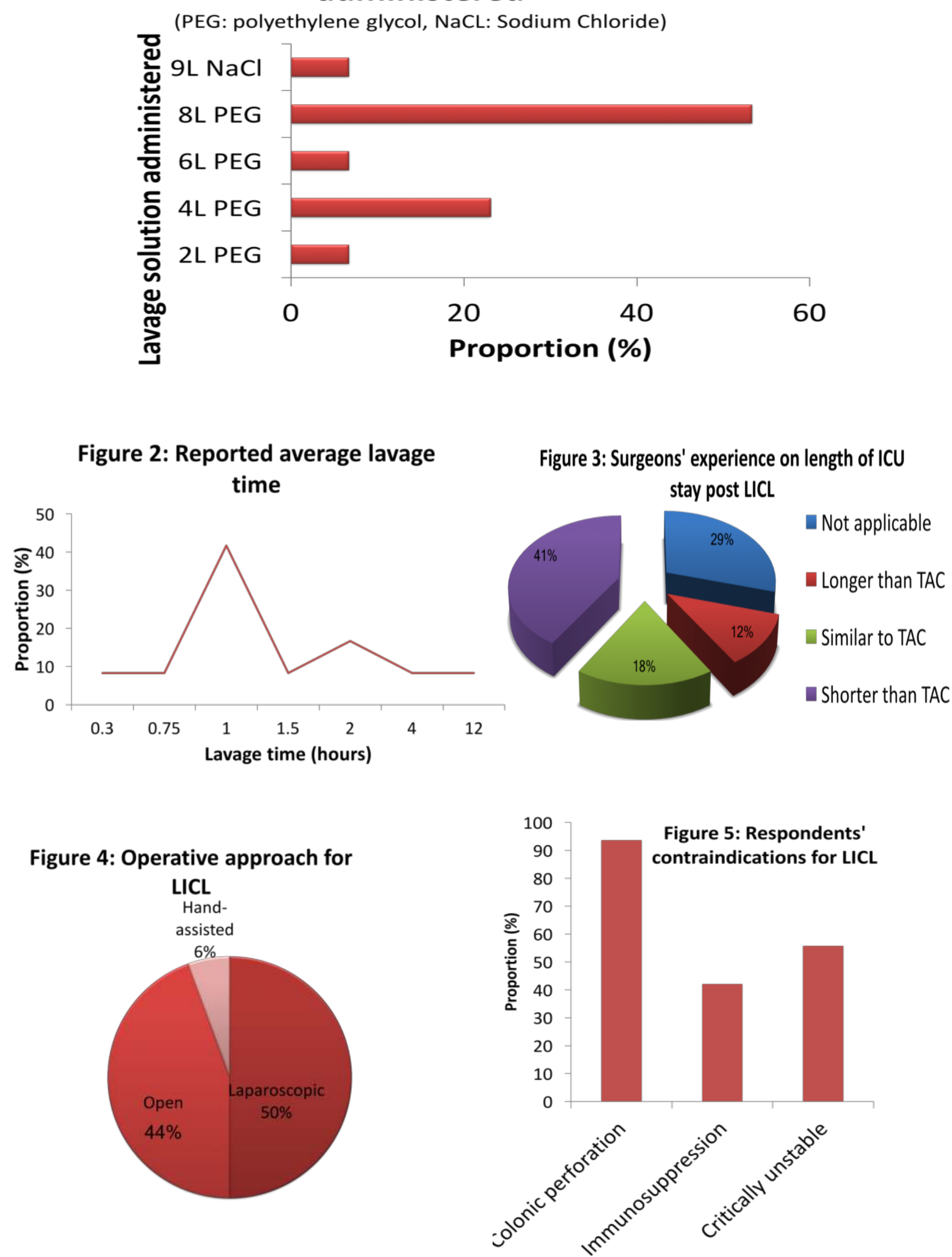

Figure 6: Loop ileostomy closure performed in at least one patient

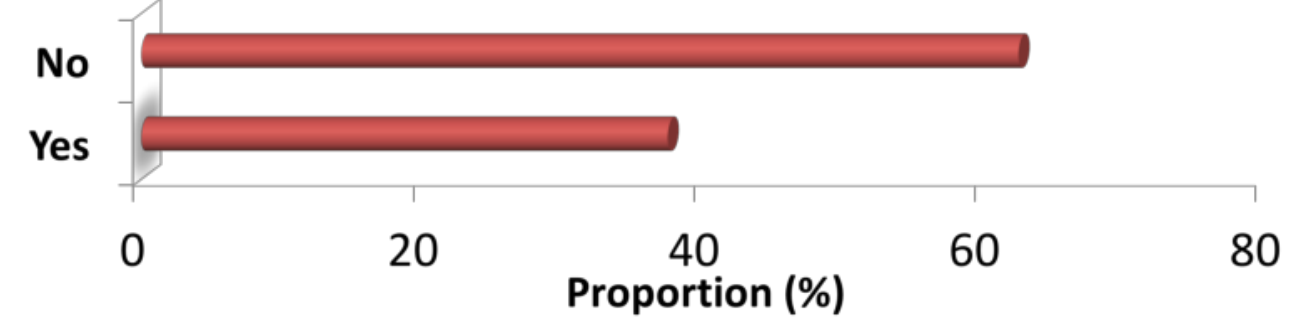

\section{Conclusion}

LICL is being used for FCDC. However, the exact indications, technique and postoperative management remain unclear and would best be determined by a prospective multicenter study. 